\title{
DIMENSIONS OF QUANTUM DETERMINANTAL RINGS
}

\author{
T. H. LENAGAN \\ Department of Mathematics, J.C.M.B., Kings Buildings, Mayfield Road, \\ Edinburgh, EH9 3JZ, Scotland, UK \\ e-mail:tom@maths.ed.ac.uk \\ and L. RIGAL \\ Université Jean Monnet (Saint-Étienne), Faculté des Sciences et Techniques, Département de \\ Mathématiques, 23 rue du Docteur Paul Michelon, 42023 Saint-Étienne Cédex 2, France \\ e-mail: laurent.rigal@univ-st-etienne.fr
}

(Received 4 September, 2000; accepted 29 January, 2001)

\begin{abstract}
We calculate the height of quantum determinantal ideals in the algebra of quantum matrices, and also the Gelfand-Kirillov, Krull and Classical Krull dimensions of the corresponding quantum determinantal factors.
\end{abstract}

2000 Mathematics Subject Classification. 16P40, 17B37, 16W35, 16P90, 16W30, 13C40, 20G42.

It has recently been shown that quantum determinantal ideals in the algebra of quantum matrices, $\mathcal{O}_{q}\left(M_{m, n}\right)$, are completely prime ideals, [3, Corollary 2.6]. In this note, we compute the height of the quantum determinantal ideals and the dimension of the corresponding quantum determinantal factors. The method is to construct a saturated chain of prime ideals that contains all of the quantum determinantal ideals. The ideals in the chain are easy to identify, the problem is to prove that they are in fact prime ideals. The method that we use to achieve this is an extension of the method used successfully in [3, Proposition 2.4] and [4, Proposition 4.4]. This method exploits the co-algebra structure of quantum matrices.

Fix a base field $k$ and choose a nonzero element $q \in k$. Given positive integers $u$, $v$, we consider the algebra of quantum matrices $\mathcal{O}_{q}\left(M_{u, v}\right)$. The computations in this paper rely on the preferred basis for the $k$-algebra $\mathcal{O}_{q}\left(M_{u, v}\right)$ introduced in [3, Section 1], and we follow the notation of that paper. See, in particular, [3, Corollary 1.11]. We recall the notation $\left[T \mid T^{\prime}\right]$ for the product of quantum minors corresponding to an allowable bitableau $\left(T, T^{\prime}\right)$. We recall also that it is sometimes convenient to label rows of $\left(T, T^{\prime}\right)$ in the form $(I, J)$ where $I$ and $J$ are sets of row and column indices, respectively (of course, $I \subseteq\{1, \ldots, u\}$ and $J \subseteq\{1, \ldots, v\}$ ); such a pair is called an index pair (see [3, Section 1]). Many of the results in [3] are stated for the square case $\mathcal{O}_{q}\left(M_{u, u}\right)$, and there are easy extensions to the rectangular case $\mathcal{O}_{q}\left(M_{u, v}\right)$, see, for example, $[3,1.11]$ and $[4$, Section 2] for more details of this standard procedure.

From now on, we fix positive integers $m, n$. Let $t$ be a positive integer; the ideal of $\mathcal{O}_{q}\left(M_{m, n}\right)$ generated by all of the $t \times t$ quantum minors is denoted by $\mathcal{I}_{t}$ and our 
aim is to compute the dimension of the factor ring $\mathcal{O}_{q}\left(M_{m, n}\right) / \mathcal{I}_{t}$. The algebras $\mathcal{O}_{q}\left(M_{m, n}\right)$ and $\mathcal{O}_{q}\left(M_{n, m}\right)$ are isomorphic via the transpose isomorphism $\tau$ which takes $X_{i j}$ to $X_{j i}$, see [6, Proposition 3.7.1]. By [6, Lemma 4.3.1], $\tau([I \mid J])=[J \mid I]$ for each index pair $(I, J)$. It follows that $\tau\left(\mathcal{I}_{t}\right)=\mathcal{I}_{t}$, for each $t$. As a consequence, in our discussion below, we assume, without loss of generality, that $m \leq n$. Because of the quantum Laplace expansions [6, Corollary 4.4.4], the ideal $\mathcal{I}_{t}$ in $\mathcal{O}_{q}\left(M_{m, n}\right)$ contains all the $r \times r$ quantum minors for $t \leq r \leq \min \{m, n\}$. Hence, $\left[T \mid T^{\prime}\right] \in \mathcal{I}_{t}$ whenever $\left(T, T^{\prime}\right)$ is a preferred bitableau and $T$ has at least $t$ columns.

Definition. Let $u, v, t, l>0$ be integers and let $A=\mathcal{O}_{q}\left(M_{u, v}\right)$.

(i) Set $\mathcal{I}_{t}(A)$ to be the ideal of $A$ generated by $t \times t$ minors.

(ii) For $1 \leq l \leq u$, set $P_{t, l}(A)$ to be the ideal of $A$ generated by $t \times t$ minors with row indices from $u-l+1, \ldots, u$ together with all of the $(t+1) \times(t+1)$ minors. Similarly, for $1 \leq l \leq v$, set $Q_{t, l}(A)$ to be the ideal of $A$ generated by $t \times t$ minors with column indices from $1, \ldots, l$ together with all of the $(t+1) \times(t+1)$ minors.

Whenever the algebra $A$ is $\mathcal{O}_{q}\left(M_{m, n}\right)$, we will feel free to drop reference to it, and write, for example, $P_{t, l}$ for $P_{t, l}\left(\mathcal{O}_{q}\left(M_{m, n}\right)\right)$. Note that, when $t>l, P_{t, l}(A)=$ $Q_{t, l}(A)=\mathcal{I}_{t+1}(A)$ and when $t>\min \{u, v\}, \mathcal{I}_{t}(A)=(0)$; also, $P_{t, u}(A)=Q_{t, v}(A)=$ $\mathcal{I}_{t}(A)$.

Recall that an element $a$ in an algebra $A$ is said to be normal if $a A=A a$, and that a sequence of elements $a_{1}, a_{2}, \ldots, a_{s}$ in an algebra $A$ is said to be a polynormal sequence if for each $j \in\{0, \ldots, s-1\}$ the image of $a_{j+1}$ in the algebra $A / \sum_{i=1}^{j} a_{j} A$ is a normal element. If this is the case, then the ideal generated by $a_{1}, a_{2}, \ldots, a_{s}$ in $A$ is equal to $a_{1} A+a_{2} A+\ldots+a_{s} A$.

REMARK. Let $t, l$ be positive integers with $l \leq m$. Let $(I, J)$ be an index pair such that $|I|=t$ and $I \subseteq\{m-l+1, \ldots, m\}$ and let $\left(I^{\prime}, J^{\prime}\right)$ be any index pair (i.e. $I^{\prime} \subseteq\{1, \ldots, m\}$ and $\left.J^{\prime} \subseteq\{1, \ldots, n\}\right)$. Suppose that $\left(I^{\prime}, J^{\prime}\right)<(I, J)$, then, either $\left|I^{\prime}\right|>t$ or $\left|I^{\prime}\right|=t$ and $I^{\prime} \subseteq\{m-l+1, \ldots, m\}$. This is clear from the definition of the ordering on index pairs (see $[3,1.2])$.

Using [3, Corollary A.2], it follows that the quantum minors generating $P_{t, l}$ can be arranged in a polynormal sequence and

$$
P_{t, l}=\sum[I \mid J] \mathcal{O}_{q}\left(M_{m, n}\right),
$$

where either $|I|>t$ or $|I|=t$ and $I \subseteq\{m-l+1, \ldots, m\}$.

In a similar manner,

$$
Q_{t, l}=\sum[I \mid J] \mathcal{O}_{q}\left(M_{m, n}\right)
$$

where either $|J|>t$ or $|J|=t$ and $J \subseteq\{1, \ldots, l\}$.

It can easily be checked that, for any positive integer $t$, there is a morphism of algebras

$$
\theta_{t}: \mathcal{O}_{q}\left(M_{m, n}\right) \rightarrow \mathcal{O}_{q}\left(M_{m, t}\right) \otimes \mathcal{O}_{q}\left(M_{t, n}\right)
$$

given by $X_{i j} \mapsto \sum_{k=1}^{t} Y_{i k} \otimes Z_{k j}$ where we use $X, Y, Z$ to denote the generators in $\mathcal{O}_{q}\left(M_{m, n}\right), \mathcal{O}_{q}\left(M_{m, t}\right)$ and $\mathcal{O}_{q}\left(M_{t, n}\right)$, respectively. In order to prove that certain ideals in $\mathcal{O}_{q}\left(M_{m, n}\right)$ are completely prime ideals, we will show that the ideals in question are 
the inverse images under $\theta_{t}$ of certain ideals in $\mathcal{O}_{q}\left(M_{m, t}\right) \otimes \mathcal{O}_{q}\left(M_{t, n}\right)$ which we can easily demonstrate to be completely prime.

The next lemma gives $k$-bases of various ideals that we need to consider.

Lemma 1. Let $t, l, l^{\prime}$ be positive integers such that $1 \leq l \leq m$ and $1 \leq l^{\prime} \leq n$.

(a) A k-basis for $P_{t, l}$ consists of all $\left[T \mid T^{\prime}\right]$ where $\left(T, T^{\prime}\right)$ is a preferred bitableau with first row of the form $(I, J)$ where $|I|>t$ or $|I|=t$ and $I \subseteq\{m-l+1, \ldots, m\}$.

(b) A k-basis for $Q_{t, l^{\prime}}$ consists of all $\left[T \mid T^{\prime}\right]$ where $\left(T, T^{\prime}\right)$ is a preferred bitableau with first row of the form $(I, J)$ where $|J|>t$ or $|J|=t$ and $J \subseteq\left\{1, \ldots, l^{\prime}\right\}$.

(c) A k-basis for $P_{t, l}+Q_{t, l^{\prime}}$ consists of all $\left[T \mid T^{\prime}\right]$ where $\left(T, T^{\prime}\right)$ is a preferred bitableau with first row of the form $(I, J)$ where $|I|>t$ or $|I|=t$ and either $I \subseteq\{m-l+1, \ldots, m\}$ or $J \subseteq\left\{1, \ldots, l^{\prime}\right\}$.

(d) A k-basis for $P_{t, l}\left(\mathcal{O}_{q}\left(M_{m, t}\right)\right) \otimes \mathcal{O}_{q}\left(M_{t, n}\right)+\mathcal{O}_{q}\left(M_{m, t}\right) \otimes Q_{t, l^{\prime}}\left(\mathcal{O}_{q}\left(M_{t, n}\right)\right)$ consists of all pure tensors $\left[S \mid S^{\prime}\right] \otimes\left[T \mid T^{\prime}\right]$ in $\mathcal{O}_{q}\left(M_{m, t}\right) \otimes \mathcal{O}_{q}\left(M_{t, n}\right)$ where $\left(S, S^{\prime}\right)$ and $\left(T, T^{\prime}\right)$ are preferred bitableaux such that either the first row of $\left(S, S^{\prime}\right)$ has the form $(I, J)$ where $|I|=t$ and $I \subseteq\{m-l+1, \ldots, m\}$ or the first row of $\left(T, T^{\prime}\right)$ has the form $(I, J)$ where $|J|=t$ and $J \subseteq\left\{1, \ldots, l^{\prime}\right\}$.

Proof. (a) All such $\left[T \mid T^{\prime}\right]$ are in $P_{t, l}$ by definition. The remark shows that $P_{t, l}$ is spanned by terms of the form $[I \mid J]\left[R \mid R^{\prime}\right]$ where $\left(R, R^{\prime}\right)$ is a bitableau and $R$ has at most $t$ columns, and $[I \mid J]$ is such that either $|I|>t$ or $|I|=t$ and $I \subseteq\{m-l+1, \ldots, m\}$. Now, $[I \mid J]\left[R \mid R^{\prime}\right]$ can be written as a product $\left[S \mid S^{\prime}\right]$ with top row $[I \mid J]$. Applying $[\mathbf{3}$, Corollary 1.8$]$ we see that $\left[S \mid S^{\prime}\right]$ is a linear combination of products $\left[U \mid U^{\prime}\right]$ where each $\left(U, U^{\prime}\right)$ is a preferred bitableau with top row $\left(I^{\prime}, J^{\prime}\right)$ such that either $\left|I^{\prime}\right|>t$, or $\left(I^{\prime}, J^{\prime}\right) \leq(I, J)$. In either case, the preferred bitableau is a member of the putative basis.

Part (b) is proved in a similar manner, and parts (c) and (d) then follow immediately.

The algebra $\mathcal{O}_{q}\left(M_{m, t}\right) \otimes \mathcal{O}_{q}\left(M_{t, n}\right)$ has a natural $\mathbb{Z}^{m} \otimes \mathbb{Z}^{t} \otimes \mathbb{Z}^{t} \otimes \mathbb{Z}^{n}$ grading, where $Y_{i j} \otimes 1$ has degree $\left(\varepsilon_{i}, \varepsilon_{j}, 0,0\right)$ and $1 \otimes Z_{i j}$ has degree $\left(0,0, \varepsilon_{i}, \varepsilon_{j}\right)$, where $\varepsilon_{i}$, etc are elements of the standard basis in $\mathbb{Z}^{m}$, etc. For more details of such gradings, see [3, 1.5] and $[4$, Section 4]. In the sequel, the label 'homogeneous' refers to this grading.

Theorem 2. Let $t, l, l^{\prime}$ be positive integers such that $1 \leq l \leq m$ and $1 \leq l^{\prime} \leq n$. The ideals $P_{t, l}, Q_{t, l^{\prime}}$ and $P_{t, l}+Q_{t, l^{\prime}}$ are completely prime ideals in $\mathcal{O}_{q}\left(M_{m, n}\right)$.

Proof. The case $t>m$ is trivial since then, all the ideals considered are zero. If $t=1$, then the result follows from [3, Corollary 2.6]. So, we may assume that $2 \leq t \leq m$. We then have $P_{t, l}=P_{t, l}+Q_{t, t-1}$ and $Q_{t, l^{\prime}}=P_{t, t-1}+Q_{t, l^{\prime}}$, and so it is enough to prove that $P_{t, l}+Q_{t, l^{\prime}}$ is a completely prime ideal. Suppose for the moment that we have shown that the ideal $P_{t, l}\left(\mathcal{O}_{q}\left(M_{m, t}\right)\right) \otimes \mathcal{O}_{q}\left(M_{t, n}\right)+\mathcal{O}_{q}\left(M_{m, t}\right) \otimes Q_{t, l^{\prime}}\left(\mathcal{O}_{q}\left(M_{t, n}\right)\right)$ is a completely prime ideal of $\mathcal{O}_{q}\left(M_{m, t}\right) \otimes \mathcal{O}_{q}\left(M_{t, n}\right)$. If we can show that

$$
P_{t, l}+Q_{t, l^{\prime}}=\theta_{t}^{-1}\left(P_{t, l}\left(\mathcal{O}_{q}\left(M_{m, t}\right)\right) \otimes \mathcal{O}_{q}\left(M_{t, n}\right)+\mathcal{O}_{q}\left(M_{m, t}\right) \otimes Q_{t, l^{\prime}}\left(\mathcal{O}_{q}\left(M_{t, n}\right)\right),\right.
$$

it follows that $P_{t, l}+Q_{t, l^{\prime}}$ is a completely prime ideal. It is easy to check that

$$
P_{t, l}+Q_{t, l^{\prime}} \subseteq \theta_{t}^{-1}\left(P_{t, l}\left(\mathcal{O}_{q}\left(M_{m, t}\right)\right) \otimes \mathcal{O}_{q}\left(M_{t, n}\right)+\mathcal{O}_{q}\left(M_{m, t}\right) \otimes Q_{t, l^{\prime}}\left(\mathcal{O}_{q}\left(M_{t, n}\right)\right)\right.
$$


If this inclusion is proper, choose an element $x=\sum_{i=1}^{r} \alpha_{i}\left[T_{i} \mid T_{i}^{\prime}\right]$ such that

$$
x \in \theta_{t}^{-1}\left(P_{t, l}\left(\mathcal{O}_{q}\left(M_{m, t}\right)\right) \otimes \mathcal{O}_{q}\left(M_{t, n}\right)+\mathcal{O}_{q}\left(M_{m, t}\right) \otimes Q_{t, l^{\prime}}\left(\mathcal{O}_{q}\left(M_{t, n}\right)\right) \backslash P_{t, l}+Q_{t, l^{\prime}},\right.
$$

and the $\alpha_{i}$ are nonzero scalars, the $\left(T_{i}, T_{i}^{\prime}\right)$ are distinct preferred bitableaux, and each of the $T_{i}$ has at most $t$ columns. We may assume that none of the $\left[T_{i} \mid T_{i}^{\prime}\right]$ lie in $P_{t, l}+Q_{t, l^{\prime}}$.

Thus, no $\left(T_{i}, T_{i}^{\prime}\right)$ has first row of the form $(I$, ?) where $I \subseteq\{m-l+1, \ldots, m\}$ and no $\left(T_{i}, T_{i}^{\prime}\right)$ has first row of the form $(?, J)$ where $J \subseteq\left\{1, \ldots, l^{\prime}\right\}$.

Define $m$-tuples $\bar{\rho}\left(T_{i}\right)$ as in $[3,2.2]$, and let $\bar{\rho}$ be the minimum of the $\bar{\rho}\left(T_{i}\right)$ under reverse lexicographic order. After re-indexing, we may assume that there is some $r^{\prime}$ such that $\bar{\rho}\left(T_{i}\right)=\bar{\rho}$ for $i \leq r^{\prime}$ and $\bar{\rho}\left(T_{i}\right)>_{\text {rlex }} \bar{\rho}$ for $i>r^{\prime}$. Applying [3, Lemma 2.3] to each $\theta_{t}\left[T_{i} \mid T_{i}^{\prime}\right]$ and collecting terms, we see (using the notation of $[3,2.2]$ ) that

$$
\theta_{t}(x)=\sum_{i=1}^{r^{\prime}} \alpha_{i}\left[T_{i} \mid \mu\left(T_{i}\right)\right] \otimes\left[\mu^{\prime}\left(T_{i}\right) \mid T_{i}^{\prime}\right]+\sum_{j} X_{j} \otimes Y_{j}
$$

where the $X_{j}$ and $Y_{j}$ are homogeneous with $\bar{c}\left(X_{j}\right)=\bar{r}\left(Y_{j}\right)>_{\text {rlex }} \bar{\rho}$. We then observe (as in the proof of [3, Proposition 2.4]) that all of the $X_{j}$ belong to different homogeneous components than the $\left[T_{i} \mid \mu\left(T_{i}\right)\right]$ for $i \leq r^{\prime}$. Since $\theta_{t}(x) \in P_{t, l}\left(\mathcal{O}_{q}\left(M_{m, t}\right)\right)$ $\otimes \mathcal{O}_{q}\left(M_{t, n}\right)+\mathcal{O}_{q}\left(M_{m, t}\right) \otimes Q_{t, l^{\prime}}\left(\mathcal{O}_{q}\left(M_{t, n}\right)\right)$ and since the ideal $P_{t, l}\left(\mathcal{O}_{q}\left(M_{m, t}\right)\right) \otimes$ $\mathcal{O}_{q}\left(M_{t, n}\right)+\mathcal{O}_{q}\left(M_{m, t}\right) \otimes Q_{t, l^{\prime}}\left(\mathcal{O}_{q}\left(M_{t, n}\right)\right)$ is homogeneous, it follows that

$$
\sum_{i=1}^{r^{\prime}} \alpha_{i}\left[T_{i} \mid \mu\left(T_{i}\right)\right] \otimes\left[\mu^{\prime}\left(T_{i}\right) \mid T_{i}^{\prime}\right] \in P_{t, l}\left(\mathcal{O}_{q}\left(M_{m, t}\right)\right) \otimes \mathcal{O}_{q}\left(M_{t, n}\right)+\mathcal{O}_{q}\left(M_{m, t}\right) \otimes Q_{t, l}\left(\mathcal{O}_{q}\left(M_{t, n}\right)\right) .
$$

For $1 \leq i<j \leq r^{\prime}$, either $T_{i} \neq T_{j}$ or $T_{i}^{\prime} \neq T_{j}^{\prime}$, so $\left(T_{i}, \mu\left(T_{i}\right)\right) \neq\left(T_{j}, \mu\left(T_{j}\right)\right)$ or $\left(\mu^{\prime}\left(T_{i}\right), T_{i}^{\prime}\right) \neq\left(\mu^{\prime}\left(T_{j}\right), T_{j}^{\prime}\right)$. Because of the linear independence of the preferred products in $\mathcal{O}_{q}\left(M_{m, t}\right)$ and $\mathcal{O}_{q}\left(M_{t, n}\right)$, it follows from Lemma 1(d) that either the first row of $T_{i}$ is a subset of $\{m-l+1, \ldots, m\}$ or the first row of $T_{i}^{\prime}$ is a subset of $\left\{1, \ldots, l^{\prime}\right\}$, for $1 \leq i \leq r^{\prime}$. This contradicts our choices above, and thus

$$
P_{t, l}+Q_{t, l^{\prime}}=\theta_{t}^{-1}\left(P_{t, l}\left(\mathcal{O}_{q}\left(M_{m, t}\right)\right) \otimes \mathcal{O}_{q}\left(M_{t, n}\right)+\mathcal{O}_{q}\left(M_{m, t}\right) \otimes Q_{t, l}\left(\mathcal{O}_{q}\left(M_{t, n}\right)\right) .\right.
$$

It remains to show that the ideal

$$
P_{t, l}\left(\mathcal{O}_{q}\left(M_{m, t}\right)\right) \otimes \mathcal{O}_{q}\left(M_{t, n}\right)+\mathcal{O}_{q}\left(M_{m, t}\right) \otimes Q_{t, l^{\prime}}\left(\mathcal{O}_{q}\left(M_{t, n}\right)\right)
$$

is a completely prime ideal of $\mathcal{O}_{q}\left(M_{m, t}\right) \otimes \mathcal{O}_{q}\left(M_{t, n}\right)$. The factor algebra

$$
\frac{\mathcal{O}_{q}\left(M_{m, t}\right) \otimes \mathcal{O}_{q}\left(M_{t, n}\right)}{P_{t, l}\left(\mathcal{O}_{q}\left(M_{m, t}\right)\right) \otimes \mathcal{O}_{q}\left(M_{t, n}\right)+\mathcal{O}_{q}\left(M_{m, t}\right) \otimes Q_{t, l^{\prime}}\left(\mathcal{O}_{q}\left(M_{t, n}\right)\right)}
$$

is isomorphic to the algebra

$$
\frac{\mathcal{O}_{q}\left(M_{m, t}\right)}{P_{t, l}\left(\mathcal{O}_{q}\left(M_{m, t}\right)\right)} \otimes \frac{\mathcal{O}_{q}\left(M_{t, n}\right)}{Q_{t, l}\left(\mathcal{O}_{q}\left(M_{t, n}\right)\right)},
$$

so we need to show that this latter algebra is a domain. 
In order to clarify matters, we will denote the generators of $\mathcal{O}_{q}\left(M_{m, t}\right)$ by $Y_{i j}$ and the generators of $\mathcal{O}_{q}\left(M_{t, n}\right)$ by $Z_{i j}$. Set $B$ to be the $l \times t$ quantum matrix algebra generated by the $Y_{i j}$ in $\mathcal{O}_{q}\left(M_{m, t}\right)$ with $i \geq m-l+1$. Note that $P_{t, l}(B)=\mathcal{I}_{t}(B)$. In a similar way, we set $C$ to be the $t \times l^{\prime}$ quantum matrix algebra generated by the $Z_{i j}$ with $1 \leq j \leq l^{\prime}$ then $Q_{t, l^{\prime}}(C)=\mathcal{I}_{t}(C)$.

The algebra $\mathcal{O}_{q}\left(M_{m, t}\right)$ is an Ore extension of the subalgebra $B$, with the remaining variables added in the order $Y_{m-l, t}, Y_{m-l, t-1}, \ldots, Y_{m-l, 1}, \ldots$, $Y_{1, t}, \ldots, Y_{1,1}$. The ideal $\mathcal{I}_{t}(B)$ is stable under the Ore extensions, since any $t \times t$ minor $[I \mid J]$ with $I \subseteq\{m-l+1, \ldots, m\} q$-commutes with any $Y_{i j}$ provided $i \leq m-l$ (see [3, A.2]). Similar remarks apply to the subalgebra $C$ of $\mathcal{O}_{q}\left(M_{t, n}\right)$.

From the remarks in the previous paragraph, it follows that the algebra

$$
\frac{\mathcal{O}_{q}\left(M_{m, t}\right)}{P_{t, l}\left(\mathcal{O}_{q}\left(M_{m, t}\right)\right)} \otimes \frac{\mathcal{O}_{q}\left(M_{t, n}\right)}{Q_{t, l}\left(\mathcal{O}_{q}\left(M_{t, n}\right)\right)},
$$

is an iterated Ore extension of the algebra

$$
B / \mathcal{I}_{t}(B) \otimes C / \mathcal{I}_{t}(C)
$$

For example, we can add the remaining variables in the order

$$
\begin{aligned}
& Y_{m-l, t} \otimes 1, Y_{m-l, t-1} \otimes 1, \ldots, Y_{m-l, 1} \otimes 1, \ldots, Y_{1, t} \otimes 1, \ldots, Y_{1,1} \otimes 1, \\
& 1 \otimes Z_{1, l+1}, 1 \otimes Z_{2, l+1}, \ldots, 1 \otimes Z_{t, l+1}, \ldots, 1 \otimes Z_{1, n}, \ldots, 1 \otimes Z_{t, n} .
\end{aligned}
$$

(Informally, we are adding the $Y$ variables row by row, from the bottom and moving from right to left in each row, and then adding the $Z$ variables column by column from the left and moving down each column.)

Thus, we only need to show that the algebra $B / \mathcal{I}_{t}(B) \otimes C / \mathcal{I}_{t}(C)$ is a domain. By [3, Theorem 2.4], we have two injective maps

$$
\begin{aligned}
& \phi_{B}: B / \mathcal{I}_{t}(B)=\mathcal{O}_{q}\left(M_{l, t}\right) / \mathcal{I}_{t}\left(\mathcal{O}_{q}\left(M_{l, t}\right)\right) \longrightarrow \mathcal{O}_{q}\left(M_{l, t-1}\right) \otimes \mathcal{O}_{q}\left(M_{t-1, t}\right), \\
& \phi_{C}: C / \mathcal{I}_{t}(C)=\mathcal{O}_{q}\left(M_{t, l^{\prime}}\right) / \mathcal{I}_{t}\left(\mathcal{O}_{q}\left(M_{t, l^{\prime}}\right)\right) \longrightarrow \mathcal{O}_{q}\left(M_{t, t-1}\right) \otimes \mathcal{O}_{q}\left(M_{t-1, l^{\prime}}\right) .
\end{aligned}
$$

(To be precise, [3, Theorem 2.4] only treats the case of $\mathcal{O}_{q}\left(M_{n, n}\right)$; the easy extension to nonsquare quantum matrices is explicitly stated in [4, Theorem 2.2].) The tensor product map $\phi_{B} \otimes \phi_{C}: B / \mathcal{I}_{t}(B) \otimes C / \mathcal{I}_{t}(C) \longrightarrow \mathcal{O}_{q}\left(M_{l, t-1}\right) \otimes \mathcal{O}_{q}\left(M_{t-1, t}\right)$ $\otimes \mathcal{O}_{q}\left(M_{t, t-1}\right) \otimes \mathcal{O}_{q}\left(M_{t-1, l^{\prime}}\right)$ is also injective, since the tensor products are over the base field $k$. However, the target algebra is an iterated Ore extension, and so a domain. Hence, $B / \mathcal{I}_{t}(B) \otimes C / \mathcal{I}_{t}(C)$ is also a domain.

We are now in a position to calculate the height of a quantum determinantal ideal and the dimension of the corresponding quantum determinantal factor algebra. There is a choice of dimensions for us to work with. For example, we could use Gelfand-Kirillov dimension, (Gabriel-Rentschler) Krull dimension, or classical Krull dimension. In fact, we shall show that these all take the same values for quantum determinantal factors. For any noetherian algebra $A$, it is known that $\mathrm{K} \operatorname{dim}(A) \geq \mathrm{Cl} . \mathrm{Kdim}(A)$ and that $\mathrm{GK} \operatorname{dim}(\mathrm{A}) \geq \mathrm{Cl}$. $\mathrm{Kdim}(\mathrm{A})$. In fact, for reasonably well-behaved algebras, it is $\operatorname{known}$ that $\operatorname{GKdim}(A) \geq \operatorname{Kdim}(A)$. We should clarify 
what we mean by the phrase "reasonably well-behaved algebras" in the previous sentence. If Gelfand-Kirillov dimension is known to be partitive and take only integer values on the category of finitely generated modules over an algebra $A$ then $\operatorname{GKdim}(A) \geq \operatorname{Kdim}(A)$. That these properties hold for quantum matrices is known, see, for example, [5] or [1]. Another key property that we need is that in this setting, for each of the three dimensions, if $A$ is a prime noetherian algebra and $I$ is a nonzero ideal of $A$ then $\operatorname{dim}(A) \geq \operatorname{dim}(A / I)+1$.

One of the outstanding problems for quantum matrices is whether or not catenarity holds for chains of prime ideals. A closely connected question is whether Tauvel's height formula holds: in other words, if $P$ is a prime ideal, is it true that $\operatorname{GKdim}\left(\mathcal{O}_{q}\left(M_{m, n}\right) / P\right)+\operatorname{ht}(P)=\operatorname{GKdim}\left(\mathcal{O}_{q}\left(M_{m, n}\right)\right)=m n$ ? If $q$ is a root of unity, then the algebra $O_{q}\left(M_{m, n}\right)$ is affine PI. So it is catenary and Tauvel's height formula holds for all primes. However, in the general case, it is not known whether those two properties, which were conjectured in [2], still hold. The prime ideals that we have just constructed enable us to show that the determinantal ideals do indeed satisfy this height formula, as we proceed to show.

Lemma 3. Let $t \leq m=\min \{m, n\}$. Then there is a chain of primes of length $(m-t)+(n-t)+1$ between $\mathcal{I}_{t+1}$ and $\mathcal{I}_{t}$. In particular, $\operatorname{ht}\left(\mathcal{I}_{m}\right) \geq n-m+1$.

Proof. Such a chain of primes is given by

$$
\begin{aligned}
\mathcal{I}_{t+1} & =P_{t, t-1} \subseteq P_{t, t} \subseteq P_{t, t+1} \subseteq \cdots \subseteq P_{t, m-1} \subseteq P_{t, m-1}+Q_{t, t} \subseteq \cdots \subseteq P_{t, m-1} \\
& +Q_{t, n-1} \subseteq \mathcal{I}_{t} .
\end{aligned}
$$

The fact that the inclusions in those chains are proper follows from the $k$-basis descriptions of the ideals given in Lemma 1.

We can amalgamate the chains of primes produced in the last lemma to give lower bounds on the height of $\mathcal{I}_{t}$ and on the classical Krull dimension of $\mathcal{O}_{q}\left(M_{m, n}\right) / \mathcal{I}_{t}$.

Proposition 4. Let $t \leq m=\min \{m, n\}$. Then

(a) $\operatorname{ht}\left(\mathcal{I}_{t}\right) \geq(m-t+1)(n-t+1)$

(b) $\mathrm{Cl} . \operatorname{Kdim}\left(\mathcal{O}_{q}\left(M_{m, n}\right) / \mathcal{I}_{t}\right) \geq m n-(m-t+1)(n-t+1)$.

Proof. (a)

$$
\begin{aligned}
\operatorname{ht}\left(\mathcal{I}_{t}\right) & \geq \operatorname{ht}\left(\mathcal{I}_{t} / \mathcal{I}_{t+1}\right)+\operatorname{ht}\left(\mathcal{I}_{t+1} / \mathcal{I}_{t+2}\right)+\cdots+\operatorname{ht}\left(\mathcal{I}_{m-1} / \mathcal{I}_{m}\right)+\operatorname{ht}\left(\mathcal{I}_{m}\right) \\
& \geq \sum_{s=t}^{m}\{(m-s)+(n-s)+1\} \\
& =(m-t+1)(m+n+1)-2 \sum_{s=t}^{m} s \\
& =(m-t+1)(m+n+1)-\{m(m+1)-(t-1) t\} \\
& =(m-t+1)(m+n+1)-(m-t+1)(m+t) \\
& =(m-t+1)(n-t+1) .
\end{aligned}
$$


Part (b) is proved in a similar manner:

$$
\begin{aligned}
\operatorname{Cl} \operatorname{Kdim}\left(\mathcal{O}_{q}\left(M_{m, n}\right) / \mathcal{I}_{t}\right) & \geq \operatorname{ht}\left(\mathcal{I}_{1} / \mathcal{I}_{2}\right)+\ldots+\operatorname{ht}\left(\mathcal{I}_{t-1} / \mathcal{I}_{t}\right) \\
& \geq \sum_{s=1}^{t-1}\{(m-s)+(n-s)+1\} \\
& =(t-1)(m+n+1)-2 \sum_{s=1}^{t-1} s \\
& =(t-1)(m+n+1)-(t-1) t \\
& =\ldots \\
& =m n-(m-t+1)(n-t+1) .
\end{aligned}
$$

The lower bounds obtained in the previous result, and the fact that we have an upper bound of $\operatorname{GKdim}\left(\mathcal{O}_{q}\left(M_{m, n}\right)\right)=m n$ on the sum of the two numbers enable us to specify the values precisely and to show that Tauvel's height formula holds for quantum determinantal ideals.

Corollary 5. Let $t \leq \min \{m, n\}$. Then

(a) $\operatorname{ht}\left(\mathcal{I}_{t}\right)=(m-t+1)(n-t+1)$

(b) $\mathrm{Cl} \cdot \operatorname{Kdim}\left(\mathcal{O}_{q}\left(M_{m, n}\right) / \mathcal{I}_{t}\right)=\operatorname{Kdim}\left(\mathcal{O}_{q}\left(M_{m, n}\right) / \mathcal{I}_{t}\right)=\operatorname{GKdim}\left(\mathcal{O}_{q}\left(M_{m, n}\right) / \mathcal{I}_{t}\right)=$ $m n-(m-t+1)(n-t+1)$.

(c) Consequently, $\operatorname{GKdim}\left(\mathcal{O}_{q}\left(M_{m, n}\right) / \mathcal{I}_{t}\right)+\operatorname{ht}\left(\mathcal{I}_{t}\right)=\operatorname{GKdim}\left(\mathcal{O}_{q}\left(M_{m, n}\right)\right)$.

Proof. We have already observed that

$$
\operatorname{GKdim}\left(\mathcal{O}_{q}\left(M_{m, n}\right) / \mathcal{I}_{t}\right) \geq \operatorname{Kdim}\left(\mathcal{O}_{q}\left(M_{m, n}\right) / \mathcal{I}_{t}\right) \geq \mathrm{Cl} \cdot \operatorname{Kdim}\left(\mathcal{O}_{q}\left(M_{m, n}\right) / \mathcal{I}_{t}\right) .
$$

Let "dim" stand for any of these three dimensions. We know that $\operatorname{dim}\left(\mathcal{O}_{q}\left(M_{m, n}\right) / \mathcal{I}_{t}\right)+\operatorname{ht}\left(\mathcal{I}_{t}\right) \leq \operatorname{dim}\left(\mathcal{O}_{q}\left(M_{m, n}\right)\right) \leq m n$. We have the two inequalities

$$
\operatorname{dim}\left(\mathcal{O}_{q}\left(M_{m, n}\right) / \mathcal{I}_{t}\right) \geq \mathrm{Cl} \cdot \operatorname{Kdim}\left(\mathcal{O}_{q}\left(M_{m, n}\right) / \mathcal{I}_{t}\right) \geq m n-(m-t+1)(n-t+1)
$$

and $\operatorname{ht}\left(\mathcal{I}_{t}\right) \geq(m-t+1)(n-t+1)$. If either of these inequalities is strict, we get $\operatorname{dim}\left(\mathcal{O}_{q}\left(M_{m, n}\right) / \mathcal{I}_{t}\right)+\operatorname{ht}\left(\mathcal{I}_{t}\right)>m n$, a contradiction.

\section{REFERENCES}

1. J. Gómez Torrecillas and T. H. Lenagan, Poincaré series of multifiltered algebras and partitivity, J. London Math. Soc. (2) 62 (2000), 370-380.

2. K. R. Goodearl and T. H. Lenagan, Catenarity in quantum algebras, J. Pure Appl. Algebra 111 (1996), 123-142.

3. K. R. Goodearl and T. H. Lenagan, Quantum determinantal ideals, Duke Math. J. 103 (2000), 165-190.

4. K. R. Goodearl, T. H. Lenagan and L. Rigal, The first fundamental theorem of coinvariant theory for the quantum general linear group, Publ RIMS (Kyoto) 36 (2000), 269-296. 
5. J. C. McConnell, Quantum groups, filtered rings and Gelfand-Kirillov dimension in Noncommutative ring theory (Athens, Ohio, 1989), Lecture Notes in Math. No. 1448 (Springer-Verlag, 1990) 139-147.

6. B. Parshall and J.-P. Wang, Quantum linear groups, Memoirs Amer. Math. Soc. 89 (1991). 